\title{
Response from piezoelectric elements appearing immediately after collisions with silver particles
}

\section{AUTHOR(S):}

Miyachi, Takashi; Fujii, Masayuki; Hasebe, Nobuyuki; Kobayashi, Masanori; Kuraza, Genei; Nagashima, Atsushi; Nakamura, Yusuke; ... Shibata, Hiromi; Okada, Nagaya; Tou, Tonshaku

\section{CITATION:}

Miyachi, Takashi ...[et al]. Response from piezoelectric elements appearing immediately after collisions with silver particles. JOURNAL OF APPLIED PHYSICS 2005, 98(1): 014110.

\section{ISSUE DATE:}

2005-07-01

URL:

http://hdl.handle.net/2433/39715

\section{RIGHT:}

Copyright 2005 American Institute of Physics. This article may be downloaded for personal use only. Any other use requires prior permission of the author and the American Institute of Physics. 


\title{
Response from piezoelectric elements appearing immediately after collisions with silver particles
}

\author{
Takashi Miyachi, ${ }^{\text {a) }}$ Masayuki Fujii, Nobuyuki Hasebe, Masanori Kobayashi, Genei Kuraza, \\ Atsushi Nagashima, Yusuke Nakamura, Osamu Okudaira, and Naoyuki Yamashita \\ Advanced Research Institute for Science and Engineering, Waseda University, Shinjuku-ku, \\ Tokyo 169-8555, Japan \\ Ken-ichi Nogami \\ Department of Physics, Dokkyo University School of Medicine, Mibu, Tochigi 321-0293, Japan
}

Takeshi Iwai

Research Center for Nuclear Science and Technology, University of Tokyo, Tokai, Ibaraki 319-1106, Japan

Sho Sasaki

Graduate School of Science, University of Tokyo, Bunkyo-ku, Tokyo 113-0033, Japan

Hideo Ohashi

Faculty of Marine Science, Tokyo University of Marine Science and Technology, Minato-ku, Tokyo 108-8477, Japan

Sunao Hasegawa and Hajime Yano

Institute of Space and Astronautical Science, Japan Aerospace Exploration Agency, Sagamihara, Kanagawa 229-8510, Japan

Hiromi Shibata

Graduate School of Engineering, Kyoto University, Kyoto, Kyoto 606-8501, Japan

Nagaya Okada and Tonshaku Tou

Honda Electronics Co., Ltd., Toyohashi, Aichi 441-3193, Japan

(Received 29 October 2004; accepted 26 May 2005; published online 15 July 2005)

Ferroelectric lead zirconate titanate (PZT) elements were studied by directly bombarding them with hypervelocity silver particles. The mass and velocity of these hypervelocity silver particles ranged from 1 to $80 \mathrm{pg}$ and 2 to $6 \mathrm{~km} / \mathrm{s}$, respectively. This report examines the pulsed signals observed immediately after collision. The first cycle of the pulse is discussed because the information on impact is presumably recorded on the wave form. The experimental data were analyzed using the wave form that was generated immediately after collision. Consequently, the following conclusions were made: (1) the sensitivity of the element is independent of the thickness of element, (2) the pulse height is proportional to the particle momentum over the measured range, and (3) the wave form is not explicitly related to the velocity of the particles at the time of collision. The potential of a single PZT element acts as a real-time detector for hypervelocity microparticles is discussed. (c) 2005 American Institute of Physics. [DOI: 10.1063/1.1978973]

\section{INTRODUCTION}

There is currently a demand ${ }^{1}$ for the development of a real-time detector for hypervelocity particles in order to obtain security against impact damage from space debris and/or space dust. The shortage of resources available in space requires the dust detector to be as sophisticated and simple as possible. In this regard, we have been interested in lead zirconate titanate (PZT). PZT is a ferroelectric and hence a strongly piezoelectric material. The overall characteristics of PZT as a piezoelectric material are excellent as compared with those of other materials. If the detector is based on the principle of piezoelectricity, the PZT detector can be operated as an active detector without any bias voltage. This is one of the most distinguished features of the dust detector based on PZT. Since PZT is made in ceramics and a PZT-

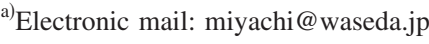

based detector has a simple structure, it can be handled without maintenance for a long duration. These attractive properties hold promise for the PZT detector to be used as a real-time detector in space in the future.

However, the PZT element is sensitive to electromagnetic and mechanical disturbances. The effects of these noises were suppressed to a millivolt level for practical applications. Therefore, it became possible to measure a signal of low amplitude.

A dust measuring tool that utilizes PZT elements incorporates the functionalities of a collector and transducer. ${ }^{2}$ The collector is the material with which hypervelocity particles collide, and strain/stress waves are generated and transmitted in it. The transducer transforms the strain into an electronic signal. So far, the PZT element has been mostly used as a transducer and not a collector. Since the particles do not strike the PZT element directly, the information regarding the impact is obtained indirectly through the process of gen- 
eration and propagation of strain/stress waves in the collector (hereafter, this detection style is referred to as the indirect method).

In addition to the indirect method, a direct method, where both the functions are integrated into a single element, is used. A few reports ${ }^{3-6}$ have discussed hypervelocity collision using the direct method. In these measurements, the output signal is processed for a period exceeding several microseconds. The signal form is then modulated by the effects of the resonant characteristics, which depend on the size and shape of the PZT element. In order to avoid a complexity in behavior, which is inherent in piezoelectric materials, we focus on a part of the output signal form, where the possible effects of interference can be neglected.

In this context, the output response immediately after collision is of particular interest to us because this part of the signal is considered to be free from external disturbances. This assumption may be confirmed by measuring the wave forms precisely in this part of the output signal before the effects of the external disturbances appear. Presumably, the wave form is determined by both the properties of piezoelectric PZT and the conditions at the time of impact. As the result, the reasons for hysteresis on impact can be unambiguously deduced to be the external disturbances including the resonance characteristics.

Therefore, we performed an experiment on the responses observed immediately after the collision using the direct method. The introduction of this new concept, referred to as the response immediately after collision, confirmed that the sensitivity of the PZT element is independent of its thickness, a fact which was not realized in the previous reports. ${ }^{5,6}$ This concept also provides an insight into the mechanism that produces the characteristic wave form and is expected to elaborate on the methods to establish a satisfactory procedure for the hypervelocity collision.

With regard to the current demand for a space-oriented application to be realized in the forthcoming decade, a PZT element is to be employed as a sensor in the dust detector onboard the Mercury Magnetospheric Orbiter of the BepiColombo project. ${ }^{7}$

In this context, it is important to report the experimental results regarding the responses observed immediately after collision. This report aims to stimulate the development of a real-time detector that is applicable to particles whose kinetic energy is as low as the order of nanojoules.

\section{EXPERIMENT}

In this experiment, three types of PZT elements were utilized. The diameter and thickness of these three types were 20 and $0.5 \mathrm{~mm}$ (sample I), 20 and $1 \mathrm{~mm}$ (sample II), and 20 and $2 \mathrm{~mm}$ (sample III). These samples were polarized along the thickness. Each element was sandwiched between a pair of silver electrodes with a thickness of several micrometers and mounted on an epoxy-resin ring, as shown in Fig. 1. Since the PZT elements were affected by the mechanical and electromagnetic noises to a large extent, the effects of these disturbances were suppressed simultaneously. The disturbance due to the mechanical noise originated pri-

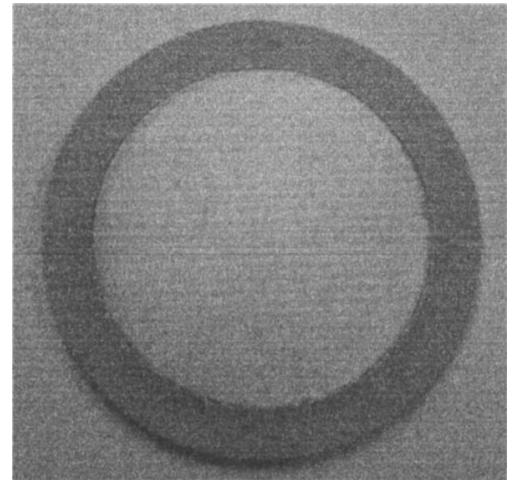

FIG. 1. PZT element. This sample is $20 \mathrm{~mm}$ in diameter and $2 \mathrm{~mm}$ in thickness. The element is supported by an epoxy-resin frame.

marily from external low-frequency vibrations. This disturbance was considerably attenuated by suspending the element using springs. The disturbance due to the electromagnetic noise was considerably attenuated by operating the amplifier using batteries. In addition, the electromagnetic noise was considerably diminished by placing the PZT element, amplifier, and batteries together in a metal box, as shown in Fig. 2. Consequently, the noise level was suppressed to a value as low as the order of millivolt. In order to avoid collisions away from the central region, a board with a hole of 10-mm diameter was placed in front of each element.

In Fig. 3, a schematic diagram of the experimental arrangement is shown. Hypervelocity silver particles were supplied by the Van de Graaff accelerator at the University of Tokyo, HIT, ${ }^{8}$ which accelerated the particles to a voltage of $U_{a}$, typically set between 2 and $2.7 \mathrm{MV}$. The particles pass through a beam line having a pair of electrodes $\left(\mathrm{T}_{1}\right.$ and $\mathrm{T}_{2}$, each with $10-\mathrm{mm}$ inner diameter and $10-\mathrm{mm}$ length) separated by a distance $L$ installed along it. The $\mathrm{T}_{1}$ and $\mathrm{T}_{2}$ pairs measure the flight time $t$ for a distance $L$, while the downstream electrode $\mathrm{T}_{2}$ measures the induced voltage $V_{0}$. Thus, the velocity $v$, electric charge $q$, and mass $m$ were obtained as $v=L / t, q=C V_{0}$, and $m=2 q U_{a} / v^{2}$, where $C$ is the feedback capacitance of $1 \mathrm{pF}$ in the amplifier. A photomultiplier tube (PMT) was placed adjacent to the PZT element in order to view the flashes of light as a time reference when the collision occurs.

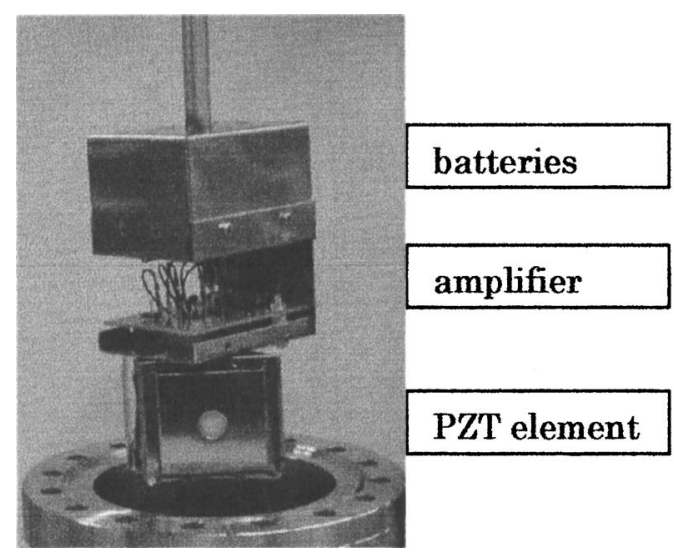

FIG. 2. Assembly of PZT element, amplifier, and batteries. In front of the PZT element, a 1-mm-thick board with a hole of $10-\mathrm{mm}$ diameter is placed. This assembly is placed in a vacuum chamber. 


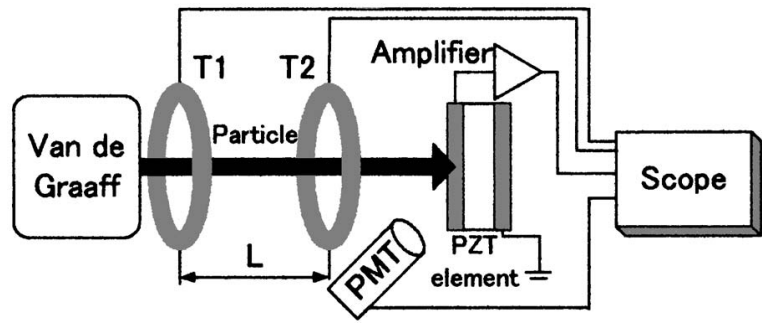

FIG. 3. Concept of the experimental arrangement. The hypervelocity particle is supplied by the Van de Graaff accelerator. The two electrodes, $\mathrm{T}_{1}$ and $\mathrm{T}_{2}$, measure the flight time between the electrodes. The induced charge is measured by $\mathrm{T}_{2}$. A photomultiplier tube (PMT) views the flashes of light at impact. The wave form is measured by a scope.

The output signal of the PZT element was processed by an amplifier with a bandwidth of $400 \mathrm{MHz}$. Its wave form was retained with the maximum possible precision, and was directed to a scope (bandwidth of $1.5 \mathrm{GHz}$ and sampling rate of $4 \mathrm{Gs} / \mathrm{s}$ ) by means of a doubly shielded cable. The wave form was digitally transformed by the scope and the data recorded from it were stored in memory. Since the time scale of the flight time was of the order of $1 \mathrm{~ms}$ and the wave form measurement was carried out with a time scale of the order of $10 \mathrm{~ns}$, the two scopes were operated simultaneously but with different time scales.

Every event was checked to determine whether the output amplitude of the PZT element exceeded a predetermined level, typically a few milivolts. When this criterion was fulfilled, this event was registered and the signals from the pair of electrodes, the PZT element and the photomultiplier tube were read. The signals of a typical event are displayed on a scope as in Fig. 4.

As described previously, the flight time between the electrodes $\mathrm{T}_{1}$ and $\mathrm{T}_{2}$ yielded the velocity of the particles $v$, and the amplitude on $\mathrm{T}_{2}$ was converted into a particle charge $q$. The signals from the PZT element and photomultiplier tube are plotted in Fig. 5 by expanding the time scale. The signal of the PZT element begins to rise in coincidence with the flashes of light, as observed using the photomultiplier tube.

A distribution of mass versus velocity is shown in Fig. 6, where the mass and velocity ranged from 1 to $80 \mathrm{pg}$ and 2 to $6 \mathrm{~km} / \mathrm{s}$, respectively.

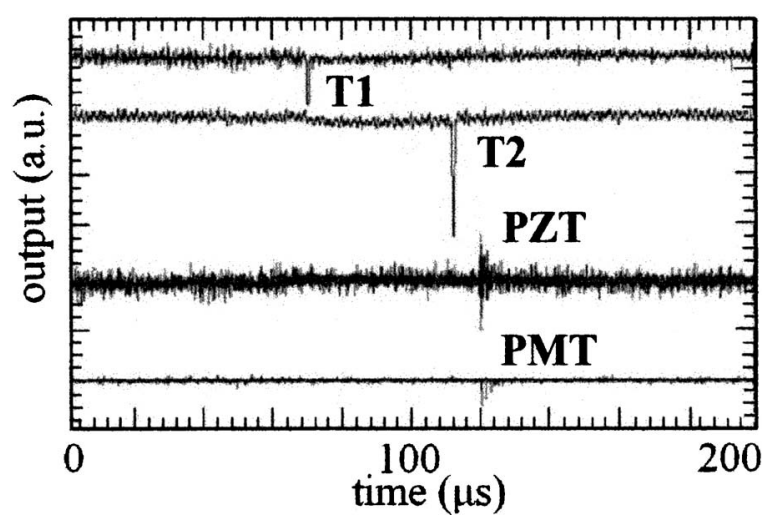

FIG. 4. Typical signal forms on the scope. From top to bottom, the signals from the electrodes $\left(\mathrm{T}_{1}\right.$ and $\left.\mathrm{T}_{2}\right)$, PZT element, and photomultiplier tube (PMT) are represented.

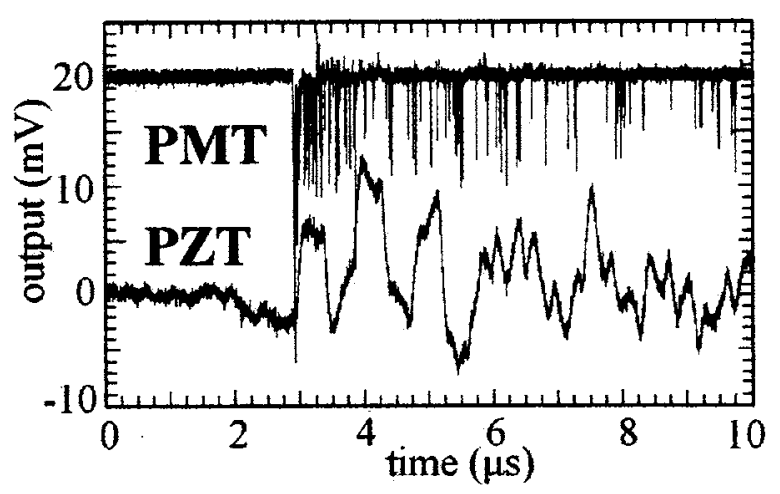

FIG. 5. Signals from the photomultiplier tube (PMT) and the PZT element. The PZT signal begins to rise in coincidence with the flash. Both signals fire in coincidence at collision. The horizontal scale is $1 \mu \mathrm{s} / \mathrm{div}$ and the vertical scale is arbitrary.

In off-line analysis, each event was visually measured for its amplitude, rise time, and the characteristics of its wave form, together with the flight time $t$ and mass $m$.

\section{RESULTS AND DISCUSSIONS}

The typical wave forms observed for samples I, II, and III, which were bombarded by silver particles having an approximate mass and velocity of $10 \mathrm{pg}$ and $5 \mathrm{~km} / \mathrm{s}$, respectively, are shown in Fig. 7. We consider a part of the first cycle of the signal forms corresponding to the samples II and III, denoted as FOC in Fig. 8. These resemble a rectangular pulse having a time period proportional to the thickness of the sample. Its amplitude is essentially constant or flat in the FOC region. In other words, the PZT element is not affected by external interactions in the FOC region. This implies that in the FOC region, the PZT element responds only to the impact. As shown in Fig. 5, the output signal starts rising in coincidence with the flashes of light. Therefore, it is considered that the flat region marked as FOC is created immediately after the collisions and is free from external disturbances primarily resulting from interference with the reflected waves.

In the case of the sample I, the signal form appears triangular. This indicates that the reflected waves arrive before the flat region has grown sufficiently. Therefore, the duration of the FOC region can be adjusted by varying the thickness of element. It appears most appropriate from a practical point of view to set the thickness of the element between 1 and $2 \mathrm{~mm}$. The output signal form appears deformed and complicated in a region that occurs after several time periods

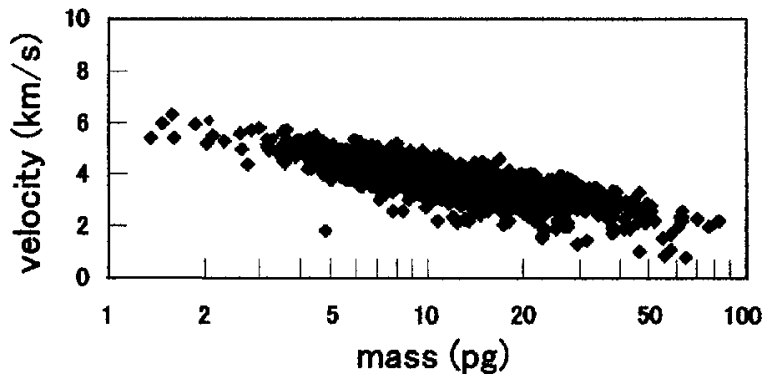

FIG. 6. Distribution of mass vs velocity of silver particles. 


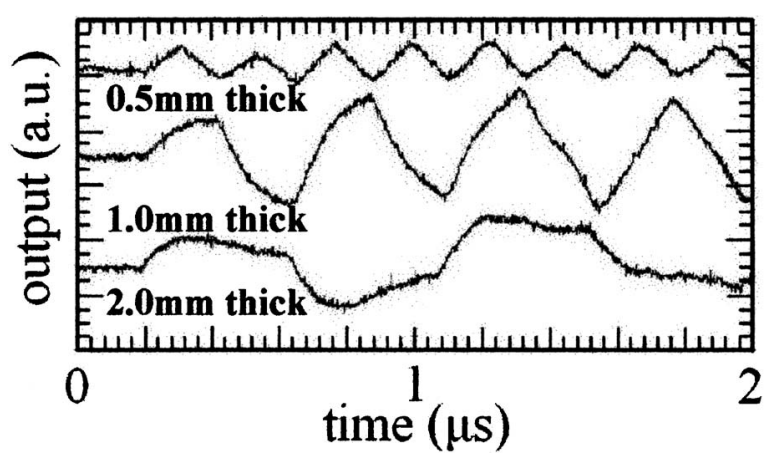

FIG. 7. Typical wave forms. From top to bottom, sample I (0.5-mm-thick element), sample II (1-mm-thick element) and sample III (2-mm-thick element). Each is the case of collision with $\sim 10-\mathrm{pg}$ silver particle at $\sim 5 \mathrm{~km} / \mathrm{s}$. The horizontal scale is $200 \mathrm{~ns} / \mathrm{div}$ and the vertical scale is $5 \mathrm{mV} / \mathrm{div}$ for every element.

have elapsed following the FOC region. This is due to interferences with several reflected waves and a damping of their amplitudes.

For our convenience, the variables corresponding to the amplitude and rise/falling time in the following discussions are defined to be as similar to those in Fig. 8, since the signal form is trapezoidal. The amplitude immediately after collision is denoted by $V_{i}$ and that at the first reflection by $V_{r}$. The rise time on impact is defined by $T_{i}$ and the falling time at the first reflection is denoted by $T_{r}$. In order to avoid visual ambiguities when reading these parameters, the amplitude $V_{p}$ is used instead of $V_{i}$ as shown in Fig. 9. In addition, another amplitude $V_{p p}$ is defined, which denotes the maximum peakto-peak voltage $e^{3,5,6}$ over several cycles.

To date, no a priori criterion has been established to determine whether $V_{p}$ or $V_{p p}$ is more fundamental. It has been proposed that the use of $V_{p}$ is more appropriate for evaluating the goodness of detector characteristics, for example, the sensitivity of the element. Let $\Delta V$ and $\Delta p$ represent the increments in amplitude and momentum, respectively. The sensitivity is then defined as a slope $S=\Delta V / \Delta p$. In Fig. 10, the correlation between the amplitude in the FOC region and momentum for sample II and III are shown. The two clusters are observed to overlap, therefore, the two slopes automatically become equal. This indicates that sensitivity is thickness independent.

On the other hand, the correlation outside the FOC re-

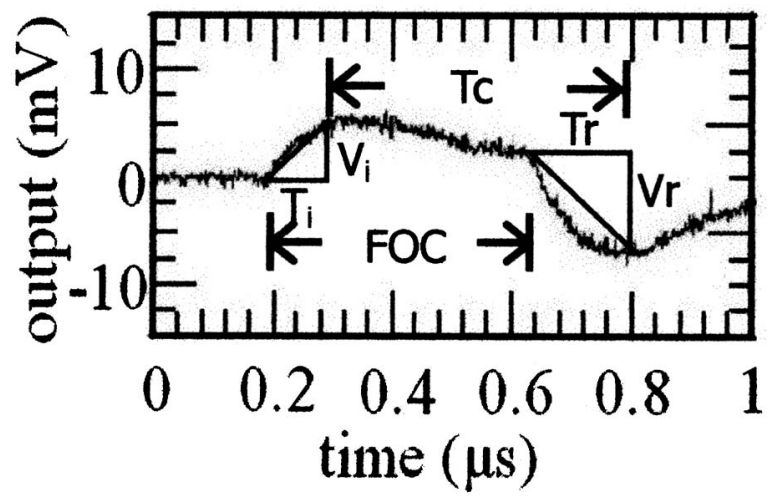

FIG. 8. Definition of variables used in the text; $V_{i}, T_{i}, V_{r}, T_{r}$, and $T_{c}$. The FOC is a region indicated by the arrow.

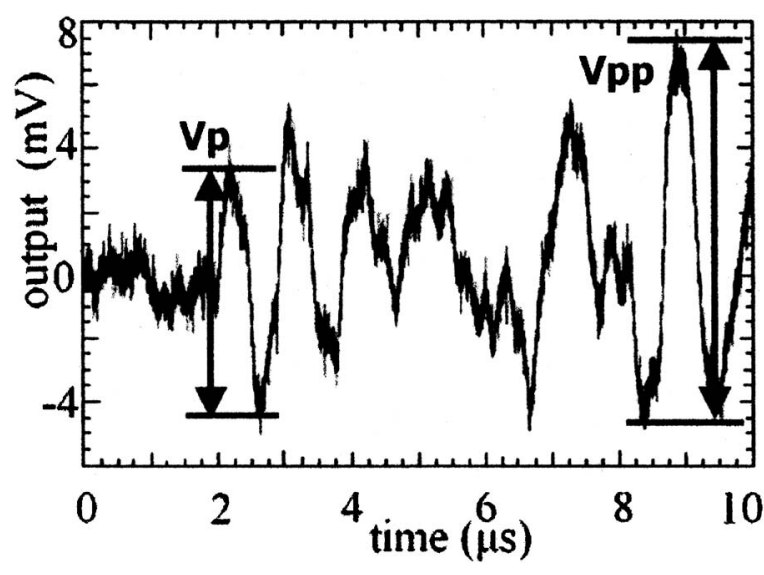

FIG. 9. Amplitudes defined in the FOC region $V_{p}$ and outside the FOC $V_{p p}$.

gion is shown in Fig. 11, in which the two clusters clearly do not overlap. In this case, it is evident that the sensitivity is thickness dependent. Therefore, the detector characteristics should be discussed based on the variables in the FOC region.

Thus, using the concept of FOC, it has been proved that the sensitivity is thickness independent. The previous reports, ${ }^{3,5,6}$ claimed that the sensitivity was thickness dependent because they did not measure the amplitude in the FOC region. This issue is resolved by introducing the concept of FOC because the thickness dependence can be explained based on the interference that occurs when several time periods have elapsed.

With regard to the rise time, $T_{i}$ is in the range of several tens nanoseconds. This time scale is comparable to that of the relaxation time ${ }^{9}$ of insulation materials compressed at $\sim 30 \mathrm{GPa}$.

In order to obtain information regarding the time period, we focus on the pulse duration $T_{c}$, denoted as "skewness" in Fig. 8 for convenience. The skewness corresponds to the time required for transmission across the thickness of the element. It is easy to determine both the edges of the skewness by visual inspection because of its characteristic form.

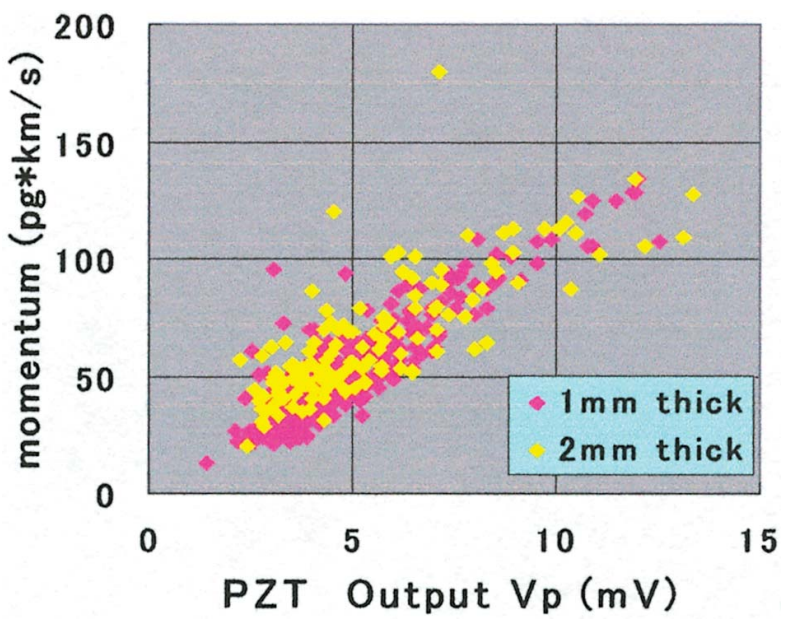

FIG. 10. (Color) Scatter plots for the output amplitude $V_{p}$ vs the momentum $p$ in the FOC region. The cluster in red is obtained with sample II and that in yellow with sample III. The two clusters overlap. The slopes $S=\Delta V / \Delta p$ representing the sensitivity of the PZT element are automatically equal. 


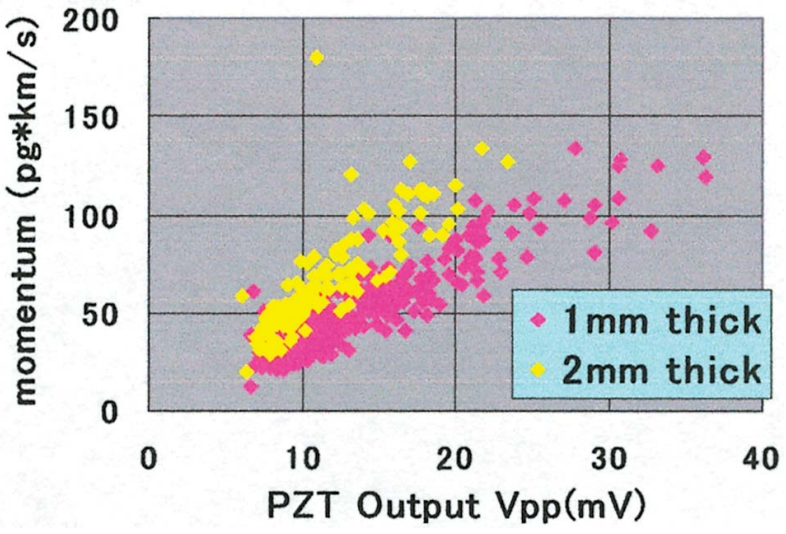

FIG. 11. (Color) Scatter plots for the output amplitude $V_{p p}$ vs the momentum $p$ outside the FOC region. The cluster in red is obtained with sample II and that in yellow with sample III. The two clusters do not overlap, consequently, the slopes of sample II and sample III are different.

Therefore, it is represented as a two-dimensional distribution in Fig. 12. It is difficult to determine a definite relationship between the skewness and the velocity at impact, however, the skewness appears contracted with increasing collision speed. However, it is not easy to confirm the relationship within the current velocity range. Therefore, it may be required to extend the available velocity range. It should be noted that the average skewness is approximately $430 \mathrm{~ns}$, and the average velocity is $4.7 \mathrm{~km} / \mathrm{s}$.

It is observed that a rapid change occurs at the first reflection, where the amplitude gap and falling time are $V_{r}$ and $T_{r}$, respectively, as shown in Fig. 8. Since it is observed that $V_{r} \sim 2 V_{i}$ and $T_{r} \sim 2 T_{i}$, the rates of change of the amplitudes are almost equal, i.e., $V_{i} / T_{i} \sim V_{r} / T_{r}$. This change is considered to be the result of a flip-flop of polarization states caused by reflection at the rear plane. This statement suggests that only a longitudinal property can participate in the reflection mechanism.

The result of Fourier analysis of sample III is shown in Fig. 13. It can be stated that the wave form decomposes into a fundamental component and a few odd harmonics because it is rectangular. The frequency components of odd harmonics suggest that the fundamental frequency would be observed at $1.1 \mathrm{MHz}$. However, a peak is known to exist at

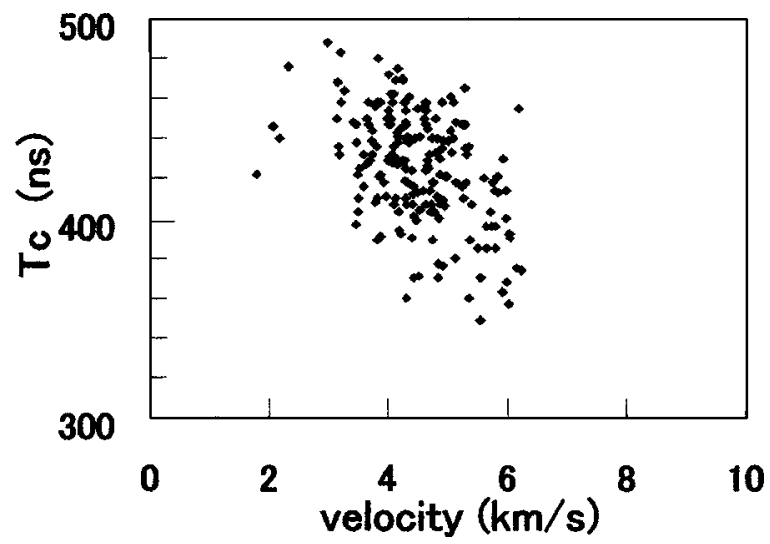

FIG. 12. Correlation between the skewness $T_{c}$ and the velocity $v$ during collision.

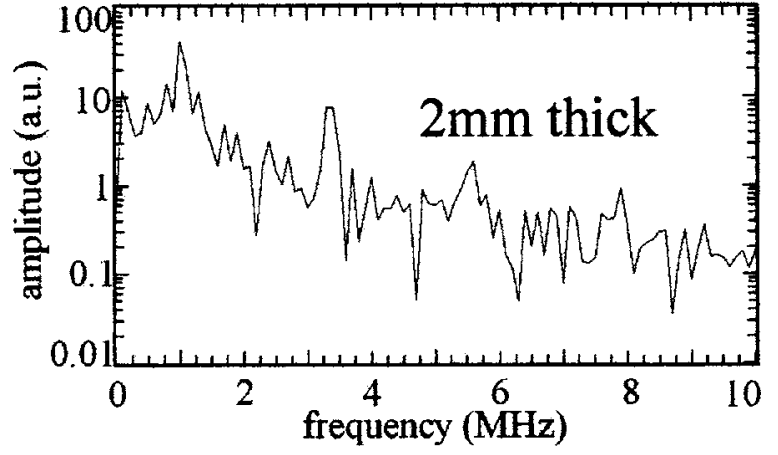

FIG. 13. Frequency components of the signal in sample III (2-mm-thick element). The odd components are dominant. Two components are involved in the peak region around $1 \mathrm{MHz}$.

$1 \mathrm{MHz}$. This indicates that a sound wave, which is sinusoidal with a frequency of approximately $1 \mathrm{MHz}$, is overlapped. Therefore, based on the simple relation between the frequency $f$, signal velocity $w$, and wavelength $\lambda, f=w / \lambda$, the velocity of the signal is $\sim 4.5 \mathrm{~km} / \mathrm{s}$ and the sound velocity is $\sim 4 \mathrm{~km} / \mathrm{s}$, provided that $\lambda / 2$ corresponds to a sample thickness of $2 \mathrm{~mm}$.

The FOC region has a flat amplitude and its duration is proportional to the thickness of the element. The flat region appears alternately in the same form over several time periods. This behavior may be explained by considering the following aspects: (1) a "charged object" moves with an approximate velocity of $4.5 \mathrm{~km} / \mathrm{s}$ within the element that is regarded as a parallel plate capacitor, ${ }^{9}$ (2) since no free charge exists within the element, there exists a "carrier," which may be a region pressurized or polarized by a shock front, moving along the thickness, and (3) the region does not stretch out over the entire volume at the instant of collision, but propagates along the thickness with a velocity of $4.5 \mathrm{~km} / \mathrm{s}$.

It is obvious that the existence of the FOC region results from cumulative processes, which have all been studied extensively. In practice, one measures average values of pressure and temperature at all microcrystal sites. Therefore, the physical states of each site could not be determined in an easy and unique manner using the results available so far. The details regarding the formation of the FOC region are open for future study.

Currently, we do not have a satisfactory procedure to understand the above results in their entirety. In addition, we have little relevant data regarding the properties of PZT at high pressures to discuss hypervelocity collisions with piezoelectric PZT material. Therefore, from a practical point of view, it is advisable to establish empirical rules by summarizing the experimental data in order to realize a hypervelocity dust detector. The output amplitude reflects on the momentum, as shown in Fig. 14. Assuming a linear relation, the momentum $p$ can be determined from the amplitude $V_{p}$ as follows:

$$
p=11 V_{p}-2 \pm \Delta p,
$$

where $p$ and $V_{p}$ are measured in $\mathrm{pg} \mathrm{km} \mathrm{s}{ }^{-1}$ and $\mathrm{mV}$, respectively, and with the ambiguity $\Delta p=10 \mathrm{pg} \mathrm{km} \mathrm{s}^{-1}$. Therefore, when establishing an empirical formula such as Eq. (1), the 


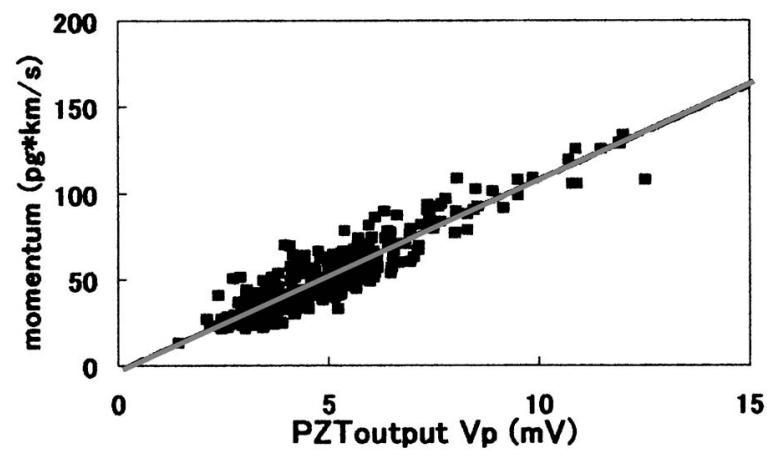

FIG. 14. Linear relationship between the output amplitudes in the FOC region and the momentum. The linear curve is obtained by a least squares fit. The solid line is represented based on Eq. (1).

momentum is uniquely obtained by considering the amplitude alone. It should be noted that this relation is independent of the thickness of the element.

Although it is difficult to evaluate the stress in PZT precisely, it is estimated using the order of magnitude based on the impedance-matched method. Let the particle velocity and (shock) wave velocity in the PZT element be represented by $u$ and $U$, respectively. The particle velocity $u$ is considered to be half that of the incident particle, i.e., $u=v / 2$, because the particle and the target densities are approximately equal. Therefore, the wave velocity $U$ is given by $U=a u+b$, where $a$ and $b$ are experimentally determined constants. Since experimental data are not available for both the constants, they are replaced by $a=(1+\Gamma) / 2$, where $\Gamma$ is the Grüneisen coefficient, and by $b=u_{0}$, which is the bulk sound speed. ${ }^{10}$ Therefore, the stress $\sigma$ is obtained to be $\sigma=\rho u U$, where $\rho$ denotes the PZT density. As shown in Fig. 15, on substituting $\rho$ $=7.5 \times 10^{3} \mathrm{~kg} / \mathrm{m}^{3}, \Gamma=2$ (Ref. 11), and $u_{0}=3 \mathrm{~km} / \mathrm{s}$ and $\sigma$ ranges from 30 to $160 \mathrm{GPa}$, partly overlapping with the previous data. ${ }^{9}$

The oscillating behavior shown in Fig. 7 is assumed to be a characteristic feature of elastic waves. Currently, we have no data on yield point of PZT. Initially, we consider the magnitude of Young's modulus as a rough estimate to distinguish between elastic and plastic states. Since the sound velocity $w_{s}$ is approximately $4 \mathrm{~km} / \mathrm{s}$, Young's modulus $E$ $=\rho w_{s}^{2}$ is $\sim 120 \mathrm{GPa}$; this value of Young's modulus corresponds to an incident velocity of approximately $5 \mathrm{~km} / \mathrm{s}$. In order to promote the use of PZT in space, it is necessary to

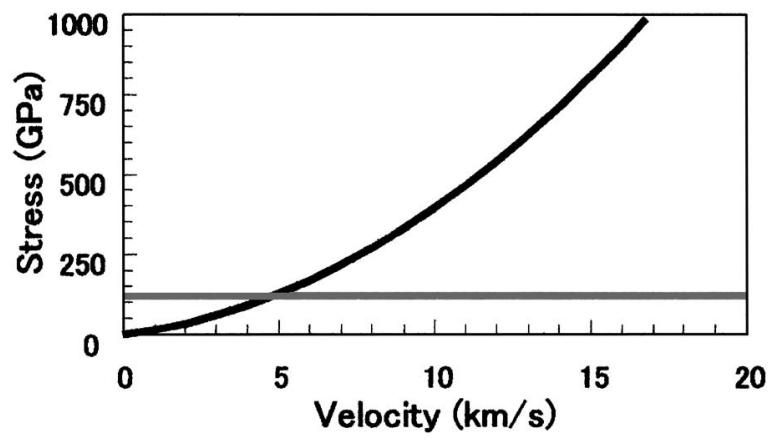

FIG. 15. Estimate of stress in the PZT element. Young's modulus is shown as a horizontal line around $120 \mathrm{GPa}$, at which the velocity during collision corresponds to about $5 \mathrm{~km} / \mathrm{s}$. study the response immediately after collision in elastic as well as plastic states by extending the collision velocity to a region with greater hypervelocity.

\section{CONCLUSION}

In this report, we discussed the response of piezoelectric PZT material by analyzing the output signal form in detail. As the result, it was found that the analysis based on the concept of FOC was essential to examine the sequence of events during collision. The sensitivity was proved to be thickness independent based on the amplitude in the FOC region, and the momentum was well described by the linear relation mentioned in Eq. (1).

The characteristic features concerning FOC can be summarized as follows: (1) A flat region of pulsed signal immediately after collision is uniquely identified as the FOC region, where the effects of external disturbances are negligible. (2) The flat region is obtained by adjusting the thickness of the PZT element. From a practical point of view, its thickness is optimized at $1-2 \mathrm{~mm}$. (3) The longitudinal wave travels at a speed of approximately $4.5 \mathrm{~km} / \mathrm{s}$ along the thickness. (4) Since no free charge substantially exists in the element, a certain type of carrier is assumed to exist. (5) Since the signal form is partially determined based on the solid-state properties, we could identify hysteresis at impact by analyzing the wave form.

The amplitude in the FOC region increases linearly with the particle momentum, and the time response in terms of the skewness appears to decrease with the incident velocity. Therefore, the relation between the skewness $T_{c}$ or rise time $T_{i}$ and the velocity at impact $v$ has to be investigated in a wider velocity region. Once the correlation between $T_{c}\left(T_{i}\right)$, and $v$ is empirically established, it is possible to determine $m$ and $v$ individually because the momentum can be uniquely obtained from the amplitude. In order to discuss the hypervelocity collision in a systematic manner, further investigation is recommended in a region with a velocity higher than $6 \mathrm{~km} / \mathrm{s}$.

We propose that a single PZT element can be used as a real-time detector for hypervelocity particles. It is evident that it is quite ordinary as a counter. If a detailed measurement of the signal form in the FOC region can be carried out, the physical quantities of space dust/debris, for example, the momentum, mass, velocity, etc., can be determined by referring to the empirical formulas. It is recommended to carry out experiments with high precision in a region with greater hypervelocity not only for establishing the empirical rules but also for formulating a comprehensive framework to account for the total hypervelocity collision in the future.

\section{ACKNOWLEDGMENTS}

We thank T. Omata for operating HIT. This work is supported in part by the Grant in Aids from Japan Society for Promotion of Science and financial support for the groundbased experiment from Japan Aerospace Exploration Agency. 
${ }^{1}$ S. Kibe (private communication).

${ }^{2}$ J. A. M. McDonell, J. Phys. E 20, 747 (1987).

${ }^{3}$ T. Miyachi et al., in Proceedings of the 23rd International Symposium on Space Technology and Science, Matsue, 2002 (unpublished), pp. 17061710.

${ }^{4}$ H. L. K. Manning, I. H. Campbell, K. A. Nelson, and J. R. Yager, presented at Poster Session at 34th Division of Planetary Sciences meeting of American Astrophysics Society, Birmingham, Alabama, 2002 (unpublished).

${ }^{5}$ T. Miyachi et al., Jpn. J. Appl. Phys., Part 1 42, 1496 (2003).
${ }^{6}$ T. Miyachi et al., Adv. Space Res. 34, 935 (2004).

${ }^{7}$ H. Hayakawa, Y. Kasaba, H. Yamakawa, H. Ogata, and T. Mukai, Adv. Space Res. 33, 2142 (2004)

${ }^{8}$ S. Shibata et al., Radiat. Phys. Chem. 60, 277 (2001).

${ }^{9}$ G. E. Hauver, J. Appl. Phys. 36, 2113 (1965).

${ }^{10}$ T. Nicholas and R. F. Recht, in High Velocity Impact Dynamics, edited by J. A. Zukas (Wiley, New York, 1990), pp. 8-25.

${ }^{11}$ Y. B. Zel'dovich and Y. P. Raizer, in Physics of Shock Waves and HighTemperature Hydrodynamic Phenomena, edited by W. D. Hayes and R. F. Probstein (Dover, New York, 2002), p. 698. 